\title{
On the Topological Complexity of $\omega$-Languages of Non-Deterministic Petri Nets
}

\author{
Olivier Finkel \\ Equipe de Logique Mathématique \\ Institut de Mathématiques de Jussieu - Paris Rive Gauche \\ CNRS et Université Paris Diderot Paris 7 \\ UFR de Mathématiques case 7012 \\ 75205 Paris Cedex 13, France. \\ finkel@math.univ-paris-diderot.fr \\ Michał Skrzypczak* \\ Institute of Informatics \\ University of Warsaw \\ Banacha 2 \\ 02-097 Warsaw, Poland. \\ mskrzypczak@mimuw.edu.pl
}

\begin{abstract}
We show that there are $\boldsymbol{\Sigma}_{3}^{0}$-complete languages of infinite words accepted by non-deterministic Petri nets with Büchi acceptance condition, or equivalently by Büchi blind counter automata. This shows that $\omega$-languages accepted by non-deterministic Petri nets are topologically more complex than those accepted by deterministic Petri nets.
\end{abstract}

Keywords. Languages of infinite words; Petri nets; Büchi acceptance condition; Cantor space; topological complexity; Borel hierarchy, complete sets.

\section{Introduction}

The languages of infinite words, also called $\omega$-languages, accepted by finite automata were first studied by Büchi to prove the decidability of the monadic

*This author has been supported by National Science Centre grant no. DEC2012/05/N/ST6/03254. 
second order theory of one successor over the integers. Since then regular $\omega$ languages have been much studied and used for specification and verification of non-terminating systems, see $[21,20,15]$ for many results and references. The acceptance of infinite words by other finite machines, like pushdown automata, counter automata, Petri nets, Turing machines, ..., with various acceptance conditions, has also been considered, see [20, 5, 2, 9].

Since the set $\Sigma^{\omega}$ of infinite words over a finite alphabet $\Sigma$ is naturally equipped with the Cantor topology, a way to study the complexity of languages of infinite words accepted by finite machines is to study their topological complexity and firstly to locate them with regard to the Borel and the projective hierarchies $[21,19,5,13,20,18,17]$.

Every $\omega$-language accepted by a deterministic Büchi automaton is a $\mathbf{\Pi}_{2}^{0}$-set. On the other hand it follows from Mac Naughton's Theorem that an $\omega$-language accepted by a non-deterministic Büchi (or Muller) automaton is also accepted by a deterministic Muller automaton, and thus is a boolean combination of $\omega$-languages accepted by deterministic Büchi automata. Therefore every $\omega$-language accepted by a non-deterministic Büchi (or Muller) automaton is a $\boldsymbol{\Delta}_{3}^{0}$-set. In a similar way, every $\omega$-language accepted by a deterministic Muller Turing machine, and thus also by any Muller deterministic finite machine is a $\boldsymbol{\Delta}_{3}^{0}$-set, $[5,20]$.

We consider here acceptance of infinite words by Petri nets or equivalently by (partially) blind counter automata. Petri nets are used for the description of distributed systems $[6,16,11]$, and they may be defined as partially blind multicounter automata, as explained in $[22,5,10]$. In order to get a partially blind multicounter automaton which accepts the same language as a given Petri net, one can distinguish between the places of a Petri net by dividing them into the bounded ones (the number of tokens in such a place at any time is uniformly bounded) and the unbounded ones. Then each unbounded place may be seen as a partially blind counter, and the tokens in the bounded places determine the state of the partially blind multicounter automaton. The transitions of the Petri net may then be seen as the finite control of the partially blind multicounter automaton and the labels of these transitions are then the input symbols.

The infinite behavior of Petri nets was first studied by Valk [22] and by Carstensen in the case of deterministic Petri nets [1].

On one side the topological complexity of $\omega$-languages of deterministic Petri nets is completely determined. They are $\boldsymbol{\Delta}_{3}^{0}$-sets and their Wadge hierarchy, which is a great refinement of the Borel hierarchy, defined via reductions by continuous functions, has been determined in $[7,3,4]$; its length is the ordinal $\omega^{\omega^{2}}$.

On the other side, nothing was known about the topological complexity of $\omega$-languages of non-deterministic Petri nets. We show that there exist $\boldsymbol{\Sigma}_{3}^{0}-$ complete, hence non $\boldsymbol{\Delta}_{3}^{0}$, $\omega$-languages accepted by one-blind-counter Büchi automata. Notice that it was proved in [8] that $\omega$-languages accepted by 
(non-blind) one-counter Büchi automata have the same topological complexity as $\omega$-languages of Turing machines, but the non-blindness of the counter was essential in the proof since the ability to use the zero-test of the counter was important.

This provides the first result on the topological complexity of $\omega$-languages of non-deterministic Petri nets and shows that there exist some $\omega$-languages accepted by non-deterministic Petri nets, and even by one-blind-counter Büchi automata, which are topologically more complex than those accepted by deterministic Petri nets.

\section{Basic notions}

We assume the reader to be familiar with the theory of formal $(\omega)$-languages, see $[21,20]$.

When $\Sigma$ is a countable alphabet, a non-empty finite word over $\Sigma$ is any sequence $x=a_{1} \ldots a_{k}$, where $a_{i} \in \Sigma$ for $i=1, \ldots, k$, and $k$ is an integer $\geq 1$. $\Sigma^{\star}$ is the set of finite words (including the empty word $\varepsilon$ ) over $\Sigma$.

The first infinite ordinal is $\omega$. An $\omega$-word over $\Sigma$ is an $\omega$-sequence $a_{1} \ldots a_{n} \ldots$, where for all integers $i \geq 1, \quad a_{i} \in \Sigma$. When $\sigma$ is an $\omega$-word over $\Sigma$, we write $\sigma=\sigma(1) \sigma(2) \ldots \sigma(n) \ldots$, where for all $i, \sigma(i) \in \Sigma$, and $\sigma[n]=\sigma(1) \sigma(2) \ldots \sigma(n)$.

The concatenation product of two finite words $u$ and $v$ is denoted $u \cdot v$ and sometimes just $u v$. This product is extended to the product of a finite word $u$ and an $\omega$-word $v$ : the infinite word $u \cdot v$ is then the $\omega$-word such that: $\quad(u \cdot v)(k)=u(k)$ if $k \leq|u|$, and $(u \cdot v)(k)=v(k-|u|)$ if $k>|u|$.

The set of $\omega$-words over the alphabet $\Sigma$ is denoted by $\Sigma^{\omega}$. An $\omega$-language over an alphabet $\Sigma$ is a subset of $\Sigma^{\omega}$.

A blind multicounter automaton is a finite automaton equipped with a finite number $(k)$ of blind (sometimes called partially blind, as in [10]) counters. The content of any such counter is a non-negative integer. A counter is said to be blind when the multicounter automaton cannot test whether the content of the counter is zero. This means that if a transition of the machine is enabled when the content of a counter is zero then the same transition is also enabled when the content of the same counter is a non-zero integer.

We now give the definition of a Büchi 1-blind-counter automaton. Notice that we consider here only real time automata, i.e., without $\varepsilon$-transitions.

Definition 2.1 A (real time) Büchi 1-blind-counter automaton is a 5-tuple $\mathcal{A}=\left(Q, \Sigma, \Delta, q_{0}, F\right)$, where $Q$ is a finite set of states, $\Sigma$ is a finite input alphabet, $q_{0} \in Q$ is the initial state, the transition relation $\Delta$ is a subset of $Q \times \Sigma \times\{0,1\} \times Q \times\{0,1,-1\}$, and $F \subseteq Q$ is the set of accepting states. 
If the automaton $\mathcal{A}$ is in state $q$, and $c \in \mathbb{N}$ is the content of the counter $\mathcal{C}$, then the configuration (or global state) of $\mathcal{A}$ is the pair $(q, c)$.

Given any $a \in \Sigma$, any $q, q^{\prime} \in Q$, and any $c \in \mathbb{N}$, if both $\Delta\left(q, a, i, q^{\prime}, j\right)$, and $(c \geq 1 \Rightarrow i=1)$ and $(c=0 \Rightarrow(i=0$ and $j \in\{0,1\}))$ holds, then we write: $\quad a:(q, c) \mapsto_{\mathcal{A}}\left(q^{\prime}, c+j\right)$.

Moreover the counter of $\mathcal{A}$ is blind, i.e., if $\Delta\left(q, a, i, q^{\prime}, j\right)$ holds, and $i=0$ then $\Delta\left(q, a, i^{\prime}, q^{\prime}, j\right)$ holds also for $i^{\prime}=1$.

Let $x=a_{1} a_{2} \ldots a_{n} \ldots$ be an $\omega$-word over $\Sigma$. An $\omega$-sequence of configurations $\rho=\left(q_{i}, c_{i}\right)_{i \geq 1}$ is called a run of $\mathcal{A}$ on $x$ if and only if

- $\left(q_{1}, c_{1}\right)=\left(q_{0}, 0\right)$, and

- $a_{i}:\left(q_{i}, c_{i}\right) \mapsto_{\mathcal{A}}\left(q_{i+1}, c_{i+1}\right)$ (for all $\left.1 \leq i\right)$.

We denote In $(\rho)$ the set of all the states visited infinitely often during the run $\rho$. The automaton $\mathcal{A}$ accepts $x$ if there is an infinite run $\rho$ of $\mathcal{A}$ on $x$ such that $\operatorname{In}(\rho) \cap F \neq \emptyset$.

The $\omega$-language accepted by $\mathcal{A}$ is the set $L(\mathcal{A})$ of $\omega$-words accepted by $\mathcal{A}$.

We assume the reader to be familiar with basic notions of topology which may be found in $[14,13,12,20,15]$. If $X$ is a countable alphabet containing at least two letters, then the set $X^{\omega}$ of infinite words over $X$ may be equipped with the product topology of the discrete topology on $X$. This topology is induced by a natural metric which is called the prefix metric and defined as follows. For $u, v \in X^{\omega}$ and $u \neq v$ let $\delta(u, v)=2^{-l_{\operatorname{pref}(u, v)}}$ where $l_{\operatorname{pref}(u, v)}$ is the first integer $n$ such that the $(n+1)^{s t}$ letter of $u$ is different from the $(n+1)^{s t}$ letter of $v$.

If $X$ is finite then $X^{\omega}$ is a Cantor space and if $X$ is countably infinite then $X^{\omega}$ is homeomorphic to the Baire space $\omega^{\omega}$. The open sets of $X^{\omega}$ are the sets in the form $W \cdot X^{\omega}$, where $W \subseteq X^{\star}$.

The classes $\boldsymbol{\Sigma}_{n}^{0}$ and $\boldsymbol{\Pi}_{n}^{0}$ of the Borel Hierarchy on the topological space $X^{\omega}$ are defined as follows: $\boldsymbol{\Sigma}_{1}^{0}$ is the class of open sets of $X^{\omega}, \boldsymbol{\Pi}_{1}^{0}$ is the class of closed sets (i.e. complements of open ones) of $X^{\omega}$. And for any integer $n \geq 1$ : $\boldsymbol{\Sigma}_{n+1}^{0}$ is the class of countable unions of $\boldsymbol{\Pi}_{n}^{0}$-subsets of $X^{\omega}$, and $\boldsymbol{\Pi}_{n+1}^{0}$ is the class of countable intersections of $\boldsymbol{\Sigma}_{n}^{0}$-subsets of $X^{\omega}$. The Borel Hierarchy is also defined for transfinite levels, but we shall not need them in the present study.

Recall now the notion of completeness with regard to reduction by continuous functions. For an integer $n \geq 1$, a set $F \subseteq X^{\omega}$ is said to be a $\boldsymbol{\Sigma}_{n}^{0}$ (respectively, $\boldsymbol{\Pi}_{n}^{0}$ )-hard set iff for any set $E \subseteq Y^{\omega}$ (with $Y$ a countable alphabet): $E \in \boldsymbol{\Sigma}_{n}^{0}$ (respectively, $E \in \boldsymbol{\Pi}_{n}^{0}$ ) implies that there exists a continuous function $f: Y^{\omega} \rightarrow X^{\omega}$ such that $E=f^{-1}(F)$. If the set $F$ is $\boldsymbol{\Sigma}_{n}^{0}$ (respectively, $\boldsymbol{\Pi}_{n}^{0}$ )-hard and belongs to the class $\boldsymbol{\Sigma}_{n}^{0}$ (respectively, $\boldsymbol{\Pi}_{n}^{0}$ ) then it is said to be $\boldsymbol{\Sigma}_{n}^{0}$ (respectively, $\boldsymbol{\Pi}_{n}^{0}$ )-complete. 


\section{Topological complexity of Petri nets $\omega$-languages}

We now state and prove our main result.

Theorem 3.1 There exists a $\boldsymbol{\Sigma}_{3}^{0}$-complete $\omega$-language accepted by a Büchi one-blind-counter automaton.

The rest of this section is devoted to showing this result. First, we describe the construction of an automaton $\mathcal{A}$ recognising a $\boldsymbol{\Sigma}_{3}^{0}$-hard language. Then, in Lemma 3.2, we show that the language recognised by $\mathcal{A}$ belongs to $\Sigma_{3}^{0}$.

Let us recall an example of a $\boldsymbol{\Pi}_{3}^{0}$-complete subset $C_{3}$ of the Baire space given in [12, page 180].

$$
C_{3}=\left\{x \in \omega^{\omega} \mid \lim _{n} x(n)=\infty\right\}
$$

It follows that the set

$$
D_{3}=\left\{x \in \omega^{\omega} \mid \liminf x(n)<\infty\right\}
$$

is $\boldsymbol{\Sigma}_{3}^{0}$-complete.

Notice that we have

$D_{3}=\left\{x \in \omega^{\omega} \mid \exists N \exists^{\infty} i(x(i) \leq N)\right\}=\left\{x \in \omega^{\omega} \mid \exists N \forall p \exists i>p(x(i) \leq N)\right\}$

We now define the following coding of infinite sequences of integers by infinite words over the alphabet $\Sigma=\{a, b\}$. For $x=\left(m_{i}\right)_{i \geq 0} \in \omega^{\omega}$ we set $n_{i}=m_{i}+1$ for each $i \geq 0$, and

$$
\Phi(x)=a^{n_{0}} \cdot b^{n_{0}} \cdot a^{n_{1}} \cdot b^{n_{1}} \cdots a^{n_{i}} \cdot b^{n_{i}} \cdots
$$

It is clear that this defines a continuous injective mapping $\Phi: \omega^{\omega} \rightarrow\{a, b\}^{\omega}$. We are going to show that there exists a one-blind-counter automaton $\mathcal{A}$, reading $\omega$-words over $\Sigma$, such that $L(\mathcal{A}) \cap \Phi\left(\omega^{\omega}\right)=\Phi\left(D_{3}\right)$, what is equivalent to

$$
\forall x \in \omega^{\omega} \quad x \in D_{3} \Longleftrightarrow \Phi(x) \in L(\mathcal{A})
$$

It implies that $L(\mathcal{A})$ is $\boldsymbol{\Sigma}_{3}^{0}$-hard, since $D_{3}$ is $\boldsymbol{\Sigma}_{3}^{0}$-complete.

We start with a formal definition of $\mathcal{A}$ as depicted on Figure 1: the initial state is denoted $I$ and the unique accepting state is denoted $F$. An edge of the form $q \stackrel{a: j}{\longrightarrow} q^{\prime}$ denotes the pair of transitions $\left(q, a, 0, q^{\prime}, j\right)$ and $\left(q, a, 1, q^{\prime}, j\right)$.

For the sake of readability we use $\varepsilon$-transitions in $\mathcal{A}$. They can be eliminated in the standard way as there is no loop with only $\varepsilon$-transitions and the $\varepsilon$-transitions do not modify the counter. 


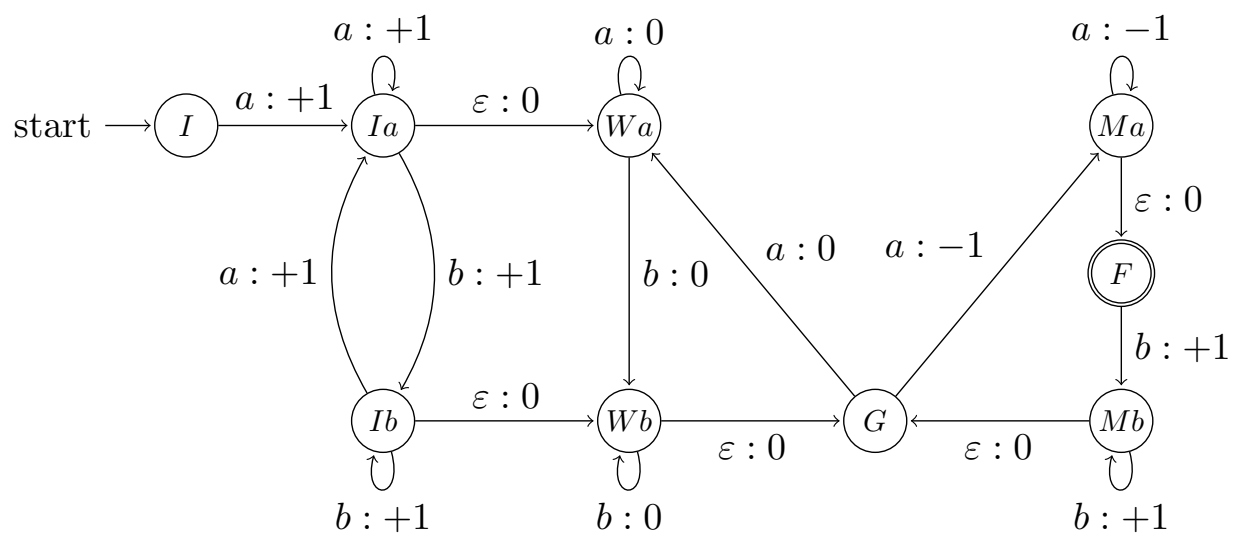

Figure 1: The automaton $\mathcal{A}$

The automaton is designed in such a way to remember the last letter read: in the states $I a, W a, M a$, and $F$ it is $a$ and in the states $I b, W b, G$, and $M b$ it is $b$.

Let $Z$ be the set of words $w \in \Sigma^{\omega}$ that contain infinitely many letters $a$ and $b$ and start from $a$. Clearly $L(\mathcal{A}) \subseteq Z$. Observe that if $w \in Z$ is an infinite word then it can be uniquely decomposed as $w=a^{n_{0}} b^{k_{0}} a^{n_{1}} b^{k_{1}} \ldots$ with $n_{i}, k_{i}>0$. A subword $a^{n_{i}} b^{k_{i}}$ of $w$ in the above decomposition is called a block of $w$.

Any accepting run of $\mathcal{A}$ on a word $w$ can be divided into three stages:

1. in the states $I, I a$, and $I b$ automaton $\mathcal{A}$ reads first $N$ letters of $w$ and increments the counter,

2. in the states $W a$ and $W b$ automaton $\mathcal{A}$ reads the rest of the current block,

3. infinitely often automaton is in $G$ at the beginning of some block $a^{n} b^{k}$ and decides either to read it without changing the counter (states $W a, W b)$ or to:

- decrease the counter on $a^{n}$ in the state $M a$,

- visit once the accepting state $F$,

- increase the counter on $b^{k}$ in $M b$.

Note that $\mathcal{A}$ can read a block $a^{n} b^{k}$ using $M a, F$, and $M b$ only if the counter value at the beginning of this block is at least $n$.

By the definition of the automaton the language recognised by $\mathcal{A}$ is the set of all words of the form $w=a^{n_{0}} b^{k_{0}} a^{n_{1}} b^{k_{1}} \ldots$ with $n_{i}, k_{i}>0$ such that for some $N \in \mathbb{N}$ and a set $I \subseteq \mathbb{N}$ we have:

- $I$ is infinite, 
- the block $a^{n_{i}} b^{k_{i}}$ for $i=\min (I)$ starts after the $N^{\prime}$ th letter of $w$,

- for every $i \in I$ we have

$$
n_{i} \leq N+\sum_{j<i \wedge j \in I} k_{j}-n_{j} .
$$

Now we want to prove (1). Let $x=\left(m_{i}\right)_{i \geq 0}$ be an element of the Baire space. Observe that $w=\Phi(x)$ has the form $a^{m_{0}+1} b^{m_{0}+1} a^{m_{1}+1} b^{m_{1}+1} \ldots$ Therefore, in the above conditions $k_{j}=n_{j}$ and (2) takes the form

$$
n_{i} \leq N \quad \text { with } \quad n_{i}=m_{i}+1 .
$$

Now, to prove (1) it is enough to observe that the following conditions are equivalent

- $\mathcal{A}$ accepts $\Phi(x)$

- there exists $N$ such that there are infinitely many $i$ with $m_{i} \leq N$

- $x \in D_{3}$.

Lemma 3.2 The language $L(\mathcal{A})$ is a $\boldsymbol{\Sigma}_{3}^{0}$ set.

Proof. First note that the set $Z$ defined above is a $\boldsymbol{\Pi}_{2}^{0}$ set, so we can restrict our attention only to words in $Z$.

Let $\rho$ be a run of $\mathcal{A}$ on a given infinite word $w=a^{n_{0}} b^{k_{0}} a^{n_{1}} b^{k_{1}} \ldots$ with $n_{i}, k_{i}>0$. We say that $\rho$ uses a block $a^{n_{i}} b^{k_{i}}$ in $w$ if $\mathcal{A}$ decreases its counter on $a^{n_{i}}$ in the state $M a$ and then increases it on $b^{k_{i}}$ in the state $M b$. We say that a block $a^{n_{i}} b^{k_{i}}$ is positive if $k_{i} \geq n_{i}$.

Now, fix a number $N$ (the guessed initial value of the counter). We describe how to inductively construct a run $\rho(N)$ of $\mathcal{A}$ on $w$. The run starts by increasing the counter $N$ times and waiting until the end of the current block. Then, when reaching the first letter of some block $a^{n} b^{k}$ with counter value $c$ in the state $G$, the following cases are possible:

- the block $a^{n} b^{k}$ is not positive then $\rho(N)$ does not use it (moves to $W a)$,

- the block is positive but $n>c$ then $\rho(N)$ does not use it (moves to $W a)$,

- the block is positive and $n \leq c$ then $\rho(N)$ uses it (moves to $M a$ ).

The following fact describes the crucial property of the run $\rho(N)$.

Fact 3.3 If $\rho$ is an accepting run of $\mathcal{A}$ on $w$ that starts with $N$ increments then $\rho(N)$ is also accepting. 
Proof. Observe that if $\rho$ is accepting then it uses infinitely many positive blocks - otherwise only finitely many non-positive ones can be used.

Now, inductively show that $\rho(N)$ has value of the counter at least equal to the value of $\rho$. In particular, whenever $\rho$ uses some positive block then $\rho(N)$ also uses it. Therefore, $\rho(N)$ uses infinitely many blocks and accepts.

Observe that given a number $N$ the condition " $\rho(N)$ uses the $i$ 'th block $a^{n_{i}} b^{k_{i}}$ " is an open property depending only on the initial segment of $w$ until the end of the block $a^{n_{i}} b^{k_{i}}$. Consider the following formula:

$$
\varphi:=\exists_{N} \forall_{i} \exists_{j \geq i} \rho(N) \text { uses the } j \text { 'th block } a^{n_{j}} b^{k_{j}} .
$$

This formula is a $\Sigma_{3}^{0}$ formula. We claim that $\varphi$ defines the language $L(\mathcal{A})$. Clearly, if a word $w$ satisfies $\varphi$ then, for the appropriate value of $N$, the run $\rho(N)$ is accepting. For the other direction, assume that $\rho$ is an accepting run of $\mathcal{A}$ on $w$. By Fact 3.3 we know that there is $N$ such that $\rho(N)$ is accepting. Therefore, $\varphi$ is satisfied on $w$.

\section{Concluding remarks}

We have proved that there are some $\boldsymbol{\Sigma}_{3}^{0}$-complete languages of infinite words accepted by blind-counter Büchi automata. This provides the first results on the topological complexity of $\omega$-languages of non-deterministic Petri nets and shows that $\omega$-languages accepted by non-deterministic Petri nets are topologically more complex than those accepted by deterministic Petri nets. A natural question is now to completely determine the Borel and Wadge hierarchies of $\omega$-languages of non-deterministic Petri nets. The first question would be: "are there non $\boldsymbol{\Sigma}_{3}^{0}$-sets accepted by non-deterministic Petri nets?".

\section{References}

[1] H. Carstensen. Infinite behaviour of deterministic Petri nets. In Proceedings of Mathematical Foundations of Computer Science 1988, volume 324 of Lecture Notes in Computer Science, pages 210-219. Springer, 1988.

[2] R.S. Cohen and A.Y. Gold. $\omega$-computations on Turing machines. Theoretical Computer Science, 6:1-23, 1978.

[3] J. Duparc, O. Finkel, and J.-P. Ressayre. The Wadge hierarchy of Petri nets $\omega$-languages. In Special Volume in Honor of Victor Selivanov at the occasion of his sixtieth birthday. Pedagogical University of Novosibirsk, 2012. Available from http://hal.archives-ouvertes.fr/hal-00743510. 
[4] J. Duparc, O. Finkel, and J.-P. Ressayre. The Wadge hierarchy of Petri nets $\omega$-languages. In Proceedings of the International Symposium on Logical Foundations of Computer Science, LFCS 2013, January 68, 2013, San Diego, California, U.S.A., Lecture Notes in Computer Science, pages 179-193. Springer, 2013. Shorter version of the preceding paper.

[5] J Engelfriet and H. J. Hoogeboom. X-automata on $\omega$-words. Theoretical Computer Science, 110(1):1-51, 1993.

[6] J. Esparza. Decidability and complexity of Petri net problems, an introduction. Lectures on Petri Nets I: Basic Models, pages 374-428, 1998.

[7] O. Finkel. An effective extension of the Wagner hierarchy to blind counter automata. In Proceedings of Computer Science Logic, 15th International Workshop, CSL 2001, volume 2142 of Lecture Notes in Computer Science, pages 369-383. Springer, 2001.

[8] O. Finkel. Borel ranks and Wadge degrees of omega context free languages. Mathematical Structures in Computer Science, 16(5):813-840, 2006 .

[9] O. Finkel. Topological complexity of context free $\omega$-languages: A survey. In Language, Culture, Computation: Studies in Honor of Yaacov Choueka, Lecture Notes in Computer Science. Springer, 2013. To appear, available from http://fr.arxiv.org/abs/0806.1413.

[10] S.A. Greibach. Remarks on blind and partially blind one way multicounter machines. Theoretical Computer Science, 7:311-324, 1978.

[11] S. Haddad. Decidability and complexity of Petri net problems. In Michel Diaz, editor, Petri Nets: Fundamental Models, Verification and Applications, pages 87-122. Wiley-ISTE, 2009.

[12] A. S. Kechris. Classical descriptive set theory. Springer-Verlag, New York, 1995.

[13] H. Lescow and W. Thomas. Logical specifications of infinite computations. In J. W. de Bakker, Willem P. de Roever, and Grzegorz Rozenberg, editors, A Decade of Concurrency, volume 803 of Lecture Notes in Computer Science, pages 583-621. Springer, 1994.

[14] Y. N. Moschovakis. Descriptive set theory. North-Holland Publishing Co., Amsterdam, 1980.

[15] D. Perrin and J.-E. Pin. Infinite words, automata, semigroups, logic and games, volume 141 of Pure and Applied Mathematics. Elsevier, 2004. 
[16] G. Rozenberg. Lectures on concurrency and Petri nets: advances in Petri nets, volume 3098. Springer Verlag, 2004.

[17] V.L. Selivanov. Fine hierarchies and m-reducibilities in theoretical computer science. Theoretical Computer Science, 405(1-2):116-163, 2008.

[18] V.L. Selivanov. Wadge reducibility and infinite computations. Special Issue on Intensional Programming and Semantics in honour of Bill Wadge on the occasion of his 60th cycle, Mathematics in Computer Science, 2(1):5-36, 2008.

[19] P. Simonnet. Automates et théorie descriptive. PhD thesis, Université Paris VII, 1992.

[20] L. Staiger. w-languages. In Handbook of formal languages, Vol. 3, pages 339-387. Springer, Berlin, 1997.

[21] W. Thomas. Automata on infinite objects. In J. van Leeuwen, editor, Handbook of Theoretical Computer Science, volume B, Formal models and semantics, pages 135-191. Elsevier, 1990.

[22] R. Valk. Infinite behaviour of Petri nets. Theoretical computer science, 25(3):311-341, 1983. 